\title{
Multiscale Evaluation of The Elastic Behavior for The Metal-Coated Lattice Structures
}

\section{Sina Soleimanian}

Xi'an Jiaotong-Liverpool University

\section{Xiang Wang}

Xi'an Jiaotong-Liverpool University

Min Chen ( $\nabla$ min.chen@xjtlu.edu.cn )

Xi'an Jiaotong-Liverpool University https://orcid.org/0000-0002-3122-6788

\section{Yanqing Yu}

Southeast University

Ji Li

Southeast University

\section{Geng Chen}

Beijing Jiaotong University

\section{Original Article}

Keywords: Metal-coated lattice, Homogenization theory, Parametric study, Specific modulus

Posted Date: October 12th, 2020

DOl: https://doi.org/10.21203/rs.3.rs-88803/v1

License: (c) (i) This work is licensed under a Creative Commons Attribution 4.0 International License. Read Full License 


\section{Title page}

\section{Multiscale evaluation of the elastic behavior for the metal-coated lattice structures}

Sina Soleimanian, born in 1991, is currently a PhD candidate of Xi' an Jiaotong-Liverpool University (XJTLU) and University of Liverpool. He received his bachelor and master degrees in Mechanical Engineering and Composite Materials Engineering both from Iran. Tel: +86-13073317304; E-mail: s.soleimanian18@student.xjtlu.edu.cn

Xiang Wang, born in 1990, is currently a PhD candidate Xi' an Jiaotong-Liverpool University (XJTLU) and University of Liverpool. He received his bachelor degree in Environmental Engineering. Tel: +86-15056935772; E-mail: xiang.wang@xjtlu.edu.cn

Min Chen, born in 1981, is currently an associate professor at Xi' an Jiaotong-Liverpool University (XJTLU).

Tel: +86-88167131; E-mail: min.chen@xjtlu.edu.cn

Yanqing Yu, born in 1995, is currently a MSc candidate at Southeast University (SEU). She received her bachelor degree in electronic science and technology.

Ji Li, born in 1983, is currently an associate professor at Southeast University (SEU). Email: j.li5@ seu.edu.cn

Geng Chen, born in 1985, is currently a professor at Beijing Jiaotong University (SEU). Email: gengchen@ bjtu.edu.cn 


\title{
ORIGINAL ARTICLE(or REVIEW)
}

\section{Multiscale evaluation of the elastic behavior for the metal-coated lattice structures}

\author{
Sina Soleimanian ${ }^{1,2 \# \bullet ~ X i a n g ~ W a n g, ~}{ }^{1,} \bullet$ Min Chen ${ }^{1 *} \bullet$ Yanqing $\mathrm{Yu}^{3} \bullet \mathrm{Ji} \mathrm{Li}^{3} \bullet$ Geng $\mathrm{Chen}^{4}$ \\ \# co-first authors * corresponding author \\ Received June xx, 201x; revised February xx, 201x; accepted March xx, 201x \\ (c) Chinese Mechanical Engineering Society and Springer-Verlag Berlin Heidelberg 2017
}

\begin{abstract}
Well-developed Additive Manufacturing leads to a variety of material and structure design. With the combination of $3 \mathrm{D}$ printing and plating technique, metal-coated resin lattice is investigated to achieve a light weight design with minimal economic cost and admirable material properties. In this paper, numerical approaches integrated with classical homogenization theory is adopted to study the effective mechanical characterizations of the BCC (Body-Centered-Cubic) metal-coated lattices. The selection of RVE (Representative Volume Element) is discussed for obtaining objective effective properties. Moreover, the impact of unit cell rod diameter and coating film thickness are investigated. A sensitivity analysis of these two parameters is conducted based on the advanced hypercube sampling methods.

The results reveal that multiple-unit-cells lead to more stable homogenized properties than single unit cell. The Increase of volume fraction may improve the elastic modulus and specific modulus remarkably. However, the increase of thickness of coating film only leads to monotonously increased elastic modulus. For this reason, there exists an optimal coating film thickness for the specific modulus of the lattice structure.
\end{abstract}

Keywords: Metal-coated lattice - Homogenization theory • Parametric study $\bullet$ Specific modulus

\section{INTRODUCTION}

Engineers interested in reducing the weight of industrial components by creating perforations for decades [1]-[3]. The idea to make perforations gives rise to lattice structure concept, which offers very promising applications nowadays. Lattices preserves the material only in necessary directions and critical zones, which can reduce the weight of the structure effectively [4].

To manufacture lattice structures, CNC milling machine can be used to make appropriate cutouts, which is limited to simple geometries and size scales [5]. In recent years, the development of Additive Manufacturing (AM) has greatly enriched the diversity of lattice structures design [6], [7] and allows to fabricate complex lattice components with uniform properties through a highly automated process [7], [9].

The input materials for $3 \mathrm{D}$ printing process can be categorized into metal and plastic types in terms of feed material. It is obvious that metallic components overwhelm the plastic ones in mechanical properties, yet the expense of 3D printing machine and base material is higher than the plastic ones. However, there are some limitations for 3D printing technique in terms of input material and desired performance for particular applications. In this regard, enhancing surfaces with metal coating film is a possible solution which can alter the material properties and attain desired characteristics. Besides the enhanced stiffness and strength, coating treatment may also lead to super specific purposes, like resistance against elevated temperatures, contact stress concentration, surface failures, fire flame, and corrosion [10]-[12]. Coating of $3 \mathrm{~d}$ printed scaffolds leads to a surface improvement which offers high cell adhesion capacity favorable for bone tissue replica [13]. Lattice structure composed of Polylactic Acid (PLA) and coated by titanium yields a compressive strength applicable for mandibular prosthesis [7]. A layer by layer coating is recommended for lattice structures so as to stimulate the bone tissue behavior and improve their biocompatibility [7]. A recent study revealed that a coating film can improve elastic modulus of BCC lattice structure up to 68 percent [14].

The general stress analysis of coated lattice structures was already studied through analytical [15], numerical [16], [17], or experimental [18] approach.

In analytical and numerical methods, lattice is treated as a special type of composites, whose equivalent properties are common to be mapped from local to global level. Multiscale evaluation of effective properties for lattice structures with narrow rods is conducted through analytical formulations in some previous studies [19]. This enables the researcher to avoid high computation cost in the simulation of large-scale structures. The bridge between local (also called RVE) and global level is the homogenization theory, which smears the structure properties through a volumetric averaging technique and mechanics constitutive laws [19]-[23].

However, most previous work in this field defined the single unit 
cell as the RVE only based on the geometry compatibility, ignoring the structural impact of the unit cell.

Moreover, few research is attributed to numerical prediction of homogenized material properties for coated lattices [24]. The rods in the plastic lattice is usually measured in millimeter, while the coating film in micrometer scale. The appropriate finite element definitions play an important role in the simulation [25], [26].

Besides for variety of design references for lattices, there are many uncertainties in the lattice printing and coating process, which involves plenty of parameters in the setting. Therefore, the major purpose of this research is to provide a reliable design reference through numerical evaluation. A BCC (body centered cubic) lattice structure is taken as an illustrative example to verify the numerical method, which was also proved by some experiments. Afterwards, two factors were studied and analyzed for their impact on the overall elastic performance of coated lattice structure.

\section{HOMOGENIZATION \\ STUDY OF LATTICE STRUCTURES}

Like conventional multi-phase composite materials, it is extremely challenging to simulate structural components composed of micro/meso-scale lattices, due to the high modelling and computational cost. Referring the classical multiscale principle, the minimal repeatable structure can be defined as the Representative Volume Element (RVE), which should be small enough not to be affected by larger scale parameters, simutaneously big enough to meet the research geometry parametrization requirement [27], [28].

\subsection{Method 1: Classical homogenization Technique}

The homogenization theory evaluates the volume weighted values for stress and strain. The core idea of this theory is that the homogenized physical properties can be interpreted as the physical parameter of the homogeneous material whose overall response is "close" to that of the heterogeneous periodic material, when the size of the cell tends to zero [20], [21]. It can be applied at micro/meso-scales satisfying the problem requirements [23].

For RVE with voids, many researchers took the effect of voids of the same size into account, although in a more complex case, two populations of voids with different sizes were studied [29]. In the present approach, the efficiency of the classic homogenization theory on prediction of effective Young's modulus is investigated when the RVE includes void regions.

According to homogenization theory, the macroscopic stress $\Sigma$ and strain $\mathrm{E}$ can be computed by integration over elemental stress $\sigma(\xi)$ and strain $\epsilon(\xi)$ as follows:

$$
\boldsymbol{\Sigma}(x)=\frac{1}{V} \int_{V} \sigma(\xi) d V
$$

Where the local coordinate $\xi$ can be referred to the lower level, and the global coordinated $x$ can be corresponded to the higher level. Stress is evaluated by volumetric averaging Eq.(1), and strain is obtained by division of secondary displacement $u$ by initial displacement $u_{0}$. Besides, $V$ is the volume of the representative element. For lattice structure,

$$
V=V_{\text {Solid }}+V_{\text {void }}
$$

while the stress of the void part is zero.

The 3D linear elastic model for material under general stress-strain condition can be given by

$$
\left\{\begin{array}{c}
\varepsilon_{x} \\
\varepsilon_{y} \\
\varepsilon_{z} \\
\gamma_{y z} \\
\gamma_{z x} \\
\gamma_{x y}
\end{array}\right\}=
$$

$$
\left[\begin{array}{cccccc}
\frac{1}{E_{x}} & -\frac{v_{y x}}{E_{y}} & -\frac{v_{z x}}{E_{z}} & \frac{1}{E_{x}} & \frac{1}{E_{x}} & \frac{1}{E_{x}} \\
-\frac{v_{x y}}{E_{x}} & \frac{1}{E_{y}} & -\frac{v_{z y}}{E_{z}} & \frac{1}{E_{x}} & \frac{1}{E_{x}} & \frac{1}{E_{x}} \\
-\frac{v_{x z}}{E_{x}} & -\frac{v_{y z}}{E_{y}} & \frac{1}{E_{z}} & \frac{1}{E_{x}} & \frac{1}{E_{x}} & \frac{1}{E_{x}} \\
\frac{1}{E_{x}} & \frac{1}{E_{x}} & \frac{1}{E_{x}} & \frac{1}{G_{y z}} & \frac{1}{E_{x}} & \frac{1}{E_{x}} \\
\frac{1}{E_{x}} & \frac{1}{E_{x}} & \frac{1}{E_{x}} & \frac{1}{E_{x}} & \frac{1}{G_{z x}} & \frac{1}{E_{x}} \\
\frac{1}{E_{x}} & \frac{1}{E_{x}} & \frac{1}{E_{x}} & \frac{1}{E_{x}} & \frac{1}{E_{x}} & \frac{1}{G_{x y}}
\end{array}\right]\left\{\begin{array}{c}
\sigma_{x} \\
\sigma_{y} \\
\sigma_{z} \\
\tau_{y z} \\
\tau_{z x} \\
\tau_{x y}
\end{array}\right\}
$$

Which can be summarized as

$$
[\varepsilon]_{6 \times 1}=[\mathrm{C}]_{6 \times 6}[\sigma]_{6 \times 1}
$$

$[C]$ is the stiffness matrix. Having a symmetric geometry, the BCC-like lattice RVE deforms isotopically; which means the properties are independent of direction. The typical directional Young's modulus for each element can be estimated as:

$$
E=\frac{\boldsymbol{\Sigma}}{\boldsymbol{E}}
$$

The efficiency of the homogenization theory can be determined if the results of method 1 are compared with another method which is expressed in the following section.

\subsection{Method 2: Hooke's Law}

Method 1 adopts the average stress-strain relations, however, there is another way to estimate the effective elastic modulus. In uniaxial tensile testing for homogeneous material, the stress can be directly determined by the reaction force and displacement. 


$$
\begin{array}{r}
\Sigma(x)=\frac{F}{A_{\text {eff }}} \\
\boldsymbol{E}(x)=\frac{\delta}{L}
\end{array}
$$

$F$ here is the reaction force caused by the input loading, $A_{e f f}$ is the cross-sectional area of the homogenized cube here. Afterwards the effective Young's modulus is computed using Eq.(6).

\subsection{Periodic boundary conditions}

Either for approach 1 or 2, the RVE should satisfy periodic conditions, which indicates that the deformed RVE under imposed loads may expand compatibly in both geometric and physical fields. There are 3 approaches in the numerical implementation, the uniform strain, the uniform stress and the periodicity approaches [30]. The first two methods are special cased and limited to symmetric geometries, while the last one is for general cases.

- Uniform strain approach (Voigt's bound)

With the assumption that the strain rate in lattice structure is uniform, the uniform strain $\boldsymbol{E}$ is imposed on $\partial V$, thus $\boldsymbol{\varepsilon}=\boldsymbol{E}$.

$$
\langle\varepsilon: D: \varepsilon\rangle=E: D^{\mathrm{eff}}: \boldsymbol{E}
$$

\section{- Uniform stress approach (Reuss's bound)}

With the assumption that the stress rate in lattice structure is uniform, the uniform stress $\boldsymbol{\Sigma}$ is imposed on $\partial V$, thus $\boldsymbol{\sigma}=\boldsymbol{\Sigma}$.

$$
\langle\sigma: C: \sigma\rangle=\Sigma: C^{\mathrm{eff}}: \Sigma
$$

$\boldsymbol{D}^{\text {eff }}$ and $\boldsymbol{C}^{\text {eff }}$ are the respective effective stiffness matrix and compliance matrix.

\section{- Periodicity approach}

The periodicity approach imposes the constraints on both fields. The stress vectors on the opposite direction have the magnitude but different sign, while the local strain can be divided into a global strain $\boldsymbol{E}$ and a fluctuating part $\boldsymbol{\varepsilon}^{\text {per }}$.

$$
\left\{\begin{array}{cc}
\boldsymbol{\sigma} \cdot \boldsymbol{n} & \text { anti - periodic } \\
\boldsymbol{\varepsilon}(u)=\boldsymbol{E}+\boldsymbol{\varepsilon}^{\text {per }} & \left\langle\boldsymbol{\varepsilon}^{\text {per }}\right\rangle=0
\end{array}\right.
$$

In this work, considering the orthogonal symmetry, the uniform strain approach is adopted, that the uniform displacement restricts the deformation of the RVE. Under this boundary condition, macroscopic stress is not uniaxial. In Method 2, the reaction force is obtained from the elastic analysis, which leads to the equivalent stress in terms to the classical stress definition.

\section{NICKEL METAL COATING}

\subsection{Coating process}

Metallic coating treatment for 3D printed resin lattice structure is studied by a research team from Southeast University [31], [32].

Metallic coating is deposited on the surface of test sample via electroless plating process which includes four major steps [31]: (1) etching, (2) sensitization/activation, (3) acceleration, and (4) plating. Test samples are first ultrasonically cleaned in isopropanol $(\mathrm{C} 3 \mathrm{H} 8 \mathrm{O})$, and then immersed in strong $\mathrm{KMnO} 4$ etchant for roughening the sample surface. After rinsing with deionized water (DI) water, the samples are sensitized/activated by dipping into a $\mathrm{Pd} / \mathrm{Sn}$ colloidal catalyst solution. Then, samples are submerged in acceleration solution for releasing Pd elementary particles. After thoroughly rinsing with DI water, the samples are immersed in alkaline nickel bath for metal plating.

\subsection{Numerical model of coating}

The coating thickness is measured in micrometer, usually in the range of $(0,25 \mu \mathrm{m})$, which is far thinner than the rod thickness of the lattice unit. The stiffness behavior of coating film is modeled by surface element with membrane and bending characteristics as.

$$
\mathrm{E}=\left[\begin{array}{cc}
k G h & 0 \\
0 & k G h
\end{array}\right]
$$

where, $k$ is the shear-correction factor, $G$ is shear modulus and $h$ is thickness of the shell.

Coating film is discretized by a surface element with membrane and bending behavior. A drawback of this type of finite element modeling is lacking the possibility to study solid-surface interaction, nonlinear elasticity. However, simplicity and ease of modeling is an advantage for surface element which cannot be attained by advanced hybrid elements.

\section{NUMERICAL RESULTS}

\subsection{Finite Element modelling}

In this work, a body-centered cubic (BCC) lattice is chosen as an investigation object. The BCC lattice structure is considered at global and RVE levels as shown by Fig. 1 (a \& b), respectively. The dimension of the unit is $3 \mathrm{~mm} \times 3 \mathrm{~mm} \times 3 \mathrm{~mm} . \eta$ is the volume fraction. Table 1 listed three lattice units with different rod diameters and their volume fractions.

$$
\eta \%=\frac{V_{\text {solid }}}{V_{\text {cube }}}
$$
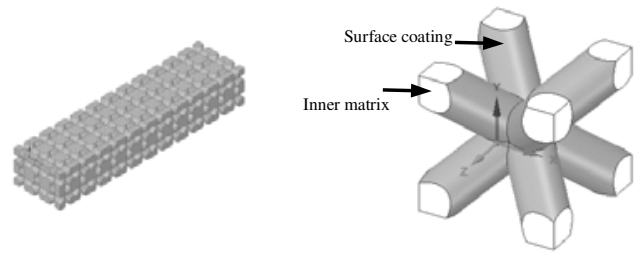

Fig. 1 BCC-like lattice structure modeling at: (a) global level, (b) RVE level. 
Table 1 Lattice unit cells with different rod diameter.

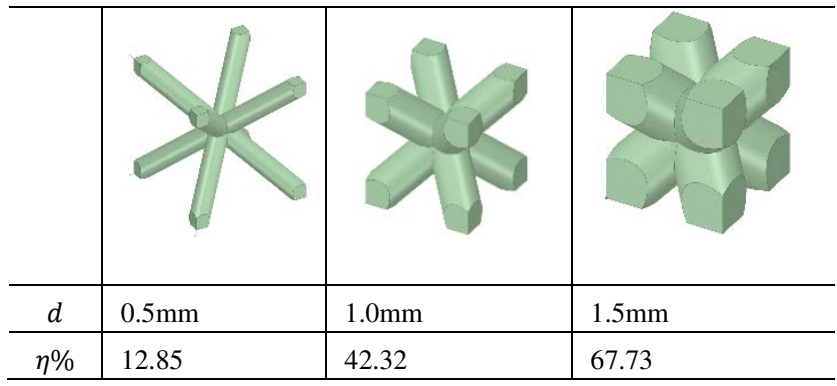

The base lattice unit is meshed by tetrahedron element using ANSYS, while its coating film is meshed through surface elements with membrane and bending behavior.

The key step is to implement the periodic boundary conditions. The configuration of lattice unit is a cube, consisting 3 pairs of opposite surfaces, noted as $\left\{A_{i}-A_{i}^{\prime}\right\}, i=1,2,3$, shown in Fig. 2.
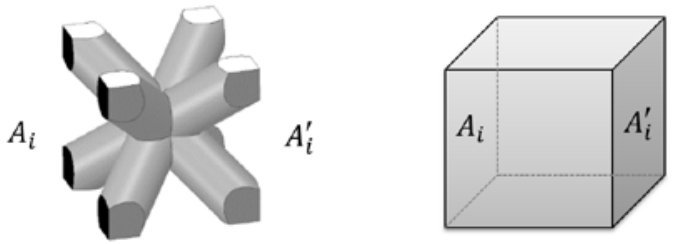

Fig. 2 Periodic boundary condition: (a) Lattice unit cell, (b) Equivalent lattice unit

The general periodic conditions are:

$$
u_{i}+u_{i}^{\prime}=U_{i}^{0}
$$

Here $U$ is the global displacement. In the BCC lattice case, $u_{i}=$ $0, u_{i}^{\prime}=U_{i}^{0}$.

The material properties of base matrix and coating film are described in Table 2.

Table 2 Material properties of base matrix and coating film

\begin{tabular}{c|c|c|c}
\hline & $\begin{array}{c}\text { Young's modulus } \\
(\mathrm{GPa})\end{array}$ & $\begin{array}{c}\text { Poisson's } \\
\text { ratio }\end{array}$ & $\begin{array}{c}\text { Density } \\
\left(\mathrm{kg} / \mathrm{m}^{3}\right)\end{array}$ \\
\hline Resin $^{1}$ & 2.46 & 0.3 & 1100 \\
\hline Coating film $^{2}$ & 50 & 0.28 & 8500 \\
\hline
\end{tabular}

1 Photosensitive resin DSM Somos 14120, from Xiamen Jiecheng 3D

Technology.

${ }^{2}$ Nickel alloy [33]

It is noteworthy to point out that both the $3 \mathrm{D}$ printing base material properties and coating film properties can be affected by many factors. The printed resin material may vary with part geometry, printing orientation, printing settings, temperature, clearing approaches and post treatments, while the coating film quality depends on settings and formulations of the coating process.

\subsection{Validation of Numerical Modeling}

To verify and compare the proposed two numerical evaluation approaches, resin matrix lattice without coating is analyzed.

- Special case: a solid cube with homogenous material A homogeneous cube was studied under the boundary condition defined in section 4.1, the effective modulus was computed based on the two proposed approach. Obviously, the exact value as the input value of the base resin, which met the theoretical evaluation.

Table 3 Effective Young's modulus evaluated by two different approach.

\begin{tabular}{c|c|c}
\hline $\begin{array}{c}\text { Initial } E \\
(\mathrm{GPa})\end{array}$ & $\begin{array}{c}\text { Approach 1: } \\
\text { Homogenization }\end{array}$ & $\begin{array}{c}\text { Approach 2: } \\
\text { Hooke's law }\end{array}$ \\
\hline 2.46 & 2.46 & 2.46 \\
\hline
\end{tabular}

- $\quad$ Literature comparison of pure lattice unit

A pure BCC lattice unit with different rod diameters were studied for the effective elastic moduli. Under the periodic boundary condition, an illustrative example of stress distribution and deformation were shown in Fig. 3. The compatibility of deformation and physical qualities are well illustrated in Fig. 4.

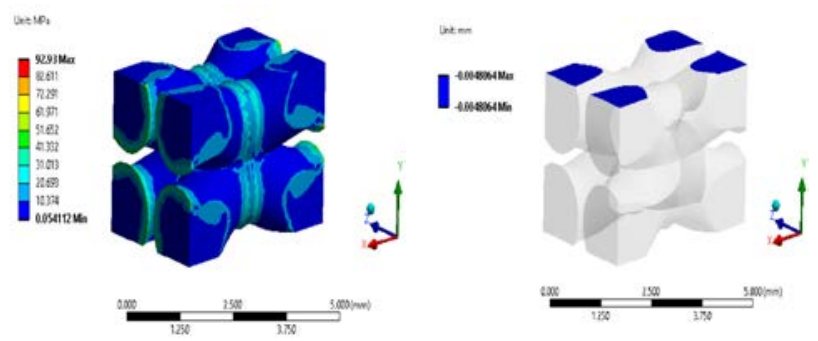

Fig. 3 (a) stress contour, and (b) transverse displacement contour for the lattice RVE

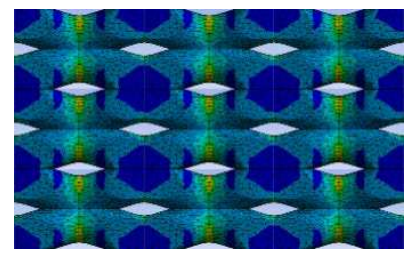

Fig. 4 Stress field of expanded deformed RVE

The obtained effective elastic modulus were compared with the result form Refai [34]. As indicated by Fig. 5, the elastic behavior versus solid volume fraction is studied and an acceptable agreement is reached for both methods, while approach 2 is more closed to the literature result.

Having a severely non-uniform spread of void leads to a nonhomogeneous distribution of mass. Consequently, the simple homogenization technique including volumetric averaging of stress has probably led to the relative bigger discrepancy.

According to the aforementioned deduction, method 2 is adopted 
for parametric studies. In general, it is recommended to use the reaction force generated by the imposed loading directly for lattice structures of all types which include a non-uniform distribution of voids.

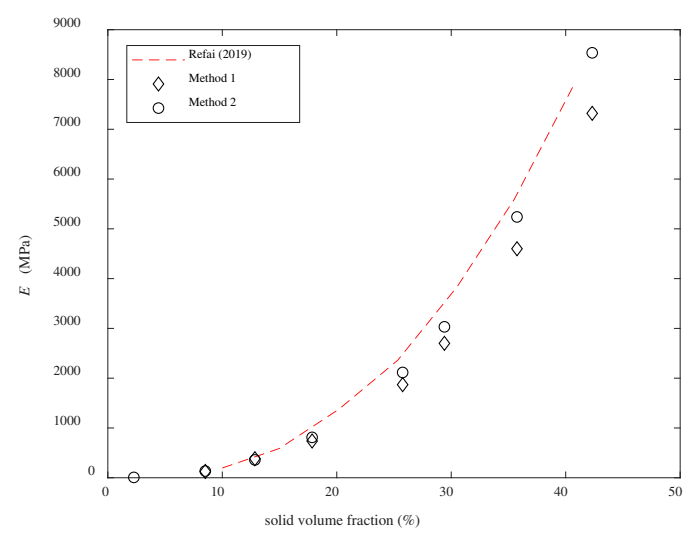

Fig. 5 Comparison of effective elastic modulus for BCC lattice RVE with various rod diameters.

\section{- Experimental validation}

To make sure that the numerical modeling simulates the structure behavior in reality, an adequate number of specimens are manufactures by $3 \mathrm{~d}$ printing technique as shown by Fig. 6).

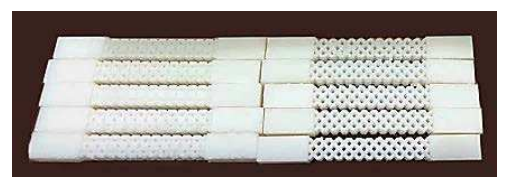

Fig. 6 3D printed lattice samples

The samples were printed by Formlabs2, with $45^{\circ}$ printing orientation, adding the thickness up to $0.05 \mathrm{~mm}$ and exposing to air for one week. Then, specimens were tested by a tensile machine with pulling rate of $2 \mathrm{~mm} / \mathrm{min}$.

As the output of tensile tests, force-displacement relationship is obtained. Subsequently, the numerically predicted homogenized elastic modulus is compared with its experimental counterpart as indicated in Fig. 7. The data shown by blue bar corresponds to the numerical result, and the bounded range colored in red refers to the range of experimental results.

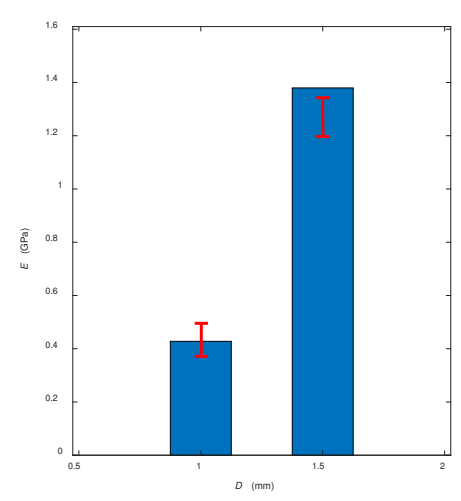

Fig. 7 Validation of numerical modeling with experiment evaluating the effective elastic modulus for lattice structure

The (min, max) error of numerical modeling with respect to experiment is achieved $(1.6,15.29 \%)$ and $(1.29,13 \%)$ for BCC lattice structure of $1 \mathrm{~mm}$ and $1.5 \mathrm{~mm}$ diameter, respectively. This error is expectable because the results of comparison between numerical RVE model and real lattice structure cannot converge due to size effect.

Overall, the experimental data for $\operatorname{rod} d=1.5 \mathrm{~mm}$ is smaller than the numerical data, which is probably affected by the washing treatment. The samples with $d=1.5 \mathrm{~mm}$ was immerged in the cleaning fluid longer time to remove the supporting materials the voids.

\subsection{Convergence of RVE response}

An RVE is defined appropriately when its homogenized properties are size independent. For the lattice structure made of uniform cells if the smallest repeatable unit can represent the homogenized material properties, the same volume monolithic structure (rectangular solid cube) should have the same properties. No matter it was expanded to other dimensions, the effective properties should be stable.

Here three types of RVE are studied, shown in Fig. 8. A single unit cell (RVE1), $2 \times 2 \times 2$ cube (RVE2) and $3 \times 3 \times 3$ cube (RVE3) are evaluated for their effective moduli with rod diameter $0.5 \mathrm{~mm}$, $1.0 \mathrm{~mm}$, and $1.5 \mathrm{~mm}$, respectively.

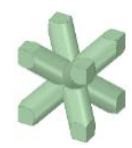

$1 \times 1 \times 1$

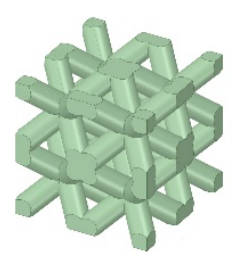

$2 \times 2 \times 2$

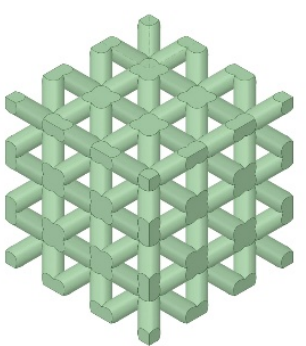

$3 \times 3 \times 3$
Fig. 8 Three RVE definitions.

The results for the effective Young's modulus are presented by the Fig. 8-Fig. 10 


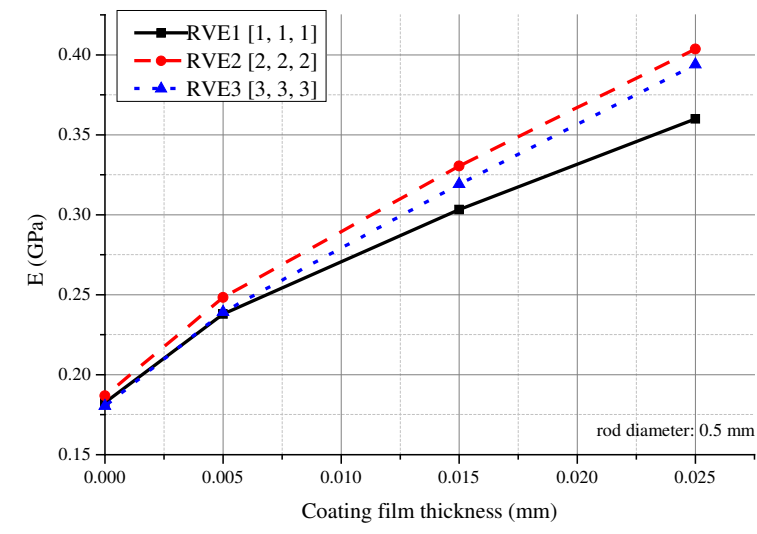

Fig. 9 Effective Young's modulus for a BCC lattice with different number of unit cells $(d=0.5 \mathrm{~mm})$

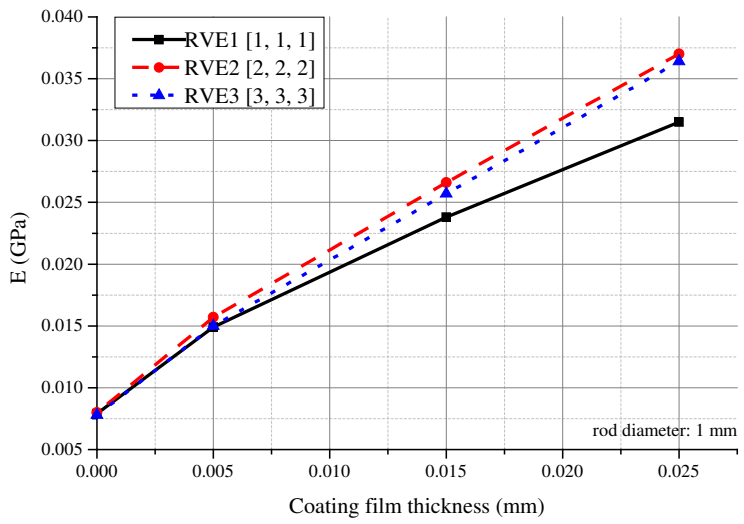

Fig. 10 Effective Young's modulus for a BCC lattice with different number of unit cells $(d=1.0 \mathrm{~mm})$

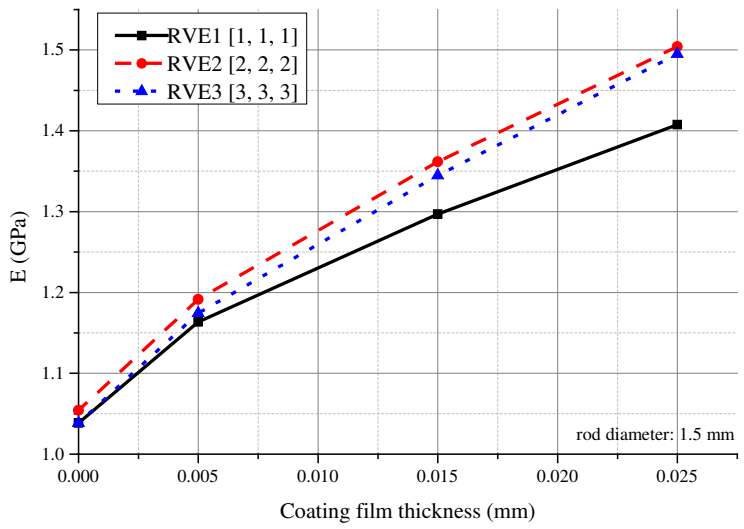

Fig. 11 Effective Young's modulus for a BCC lattice with different number of unit cells $(d=1.5 \mathrm{~mm})$

From Fig. 8, Fig. 9 and Fig. 10, it can be observed that homogenized material properties from $2 \times 2 \times 2$ and $3 \times 3 \times$ 3 cubes are quite similar, while the properties derived from single unit has relative big discrepancy with the multiple ones.

Therefore, unlikely the normal treatment in composite materials, that the minimal repeatable cell was defined as the RVE, in lattice structure, it can be concluded conservatively that the effective elastic modulus from multiple unit cells are more stable than from the single ones.

\subsection{Impact of Rod Diameter and Coating Thickness}

Although the rod diameter on elastic modulus was studied as a responsible parameter on lattice structure in a previous work [14], here the variation of elastic modulus is investigated for the coated lattice structure at RVE level.

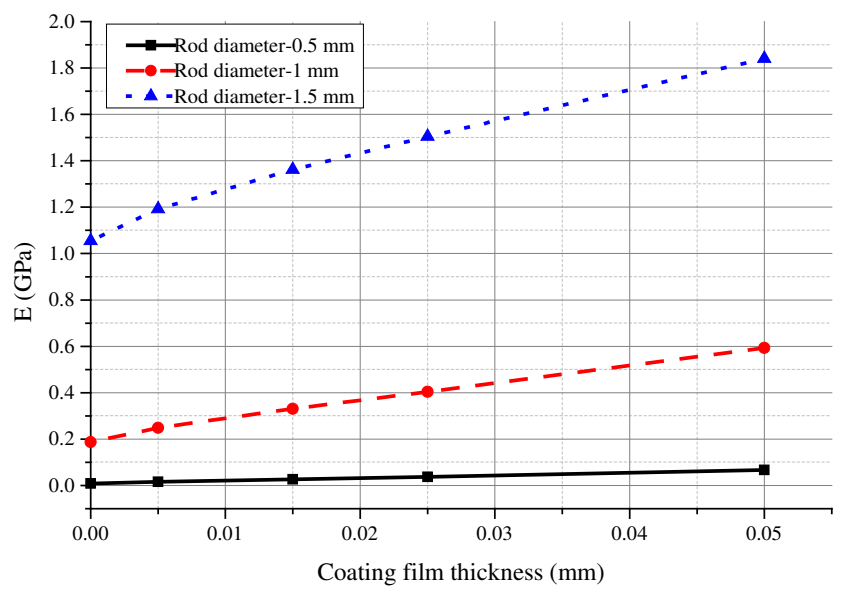

Fig. 12 Effective Young's modulus for a BCC lattice with different diameter of unit cells and coating film thickness (RVE [2,2,2] selected).

The influence of rod diameter and surface coating thickness on effective Young's modulus is indicated in Fig. 11. With the increase of rod diameter and surface coating thickness, the effective Young's modulus increases, and vice versa

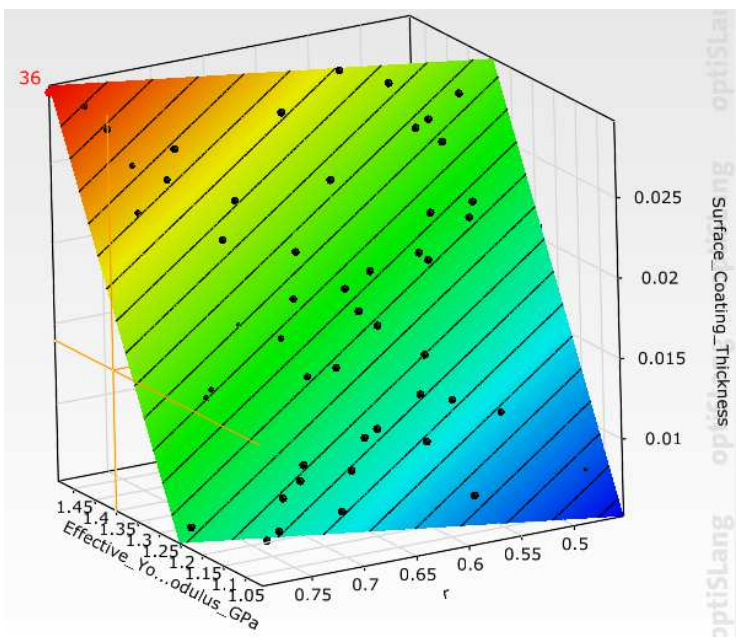

Fig. 13 Overall impact of rod radius and coating thickness on effective elastic modulus 


\subsection{Stiffness to weight ratio of metal coated lattices}

It is obvious that the coating will increase the elastic stiffness and strength of the resin lattice structures, yet the density of the coating material is much higher than the base matrix. Therefore, the overall performance, stiffness-to-weight ratio, also known as specific modulus $e$ is studied.

$$
e=\frac{E}{\rho}
$$

Here $E$ is effective Young's modulus, and $\rho$ is average density, defined as total mass over the cubic volume, instead of the solid volume. High specific modulus materials may have wide applications where minimum weight is required.

For lattice unit cells without coating, when the rod diameter is increases, the specific modulus is significantly increase accordingly as shown in Fig.14.

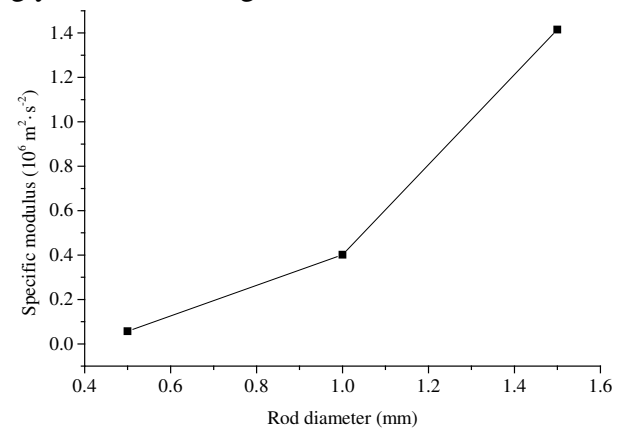

Fig. 14 Specific modulus of pure lattice units

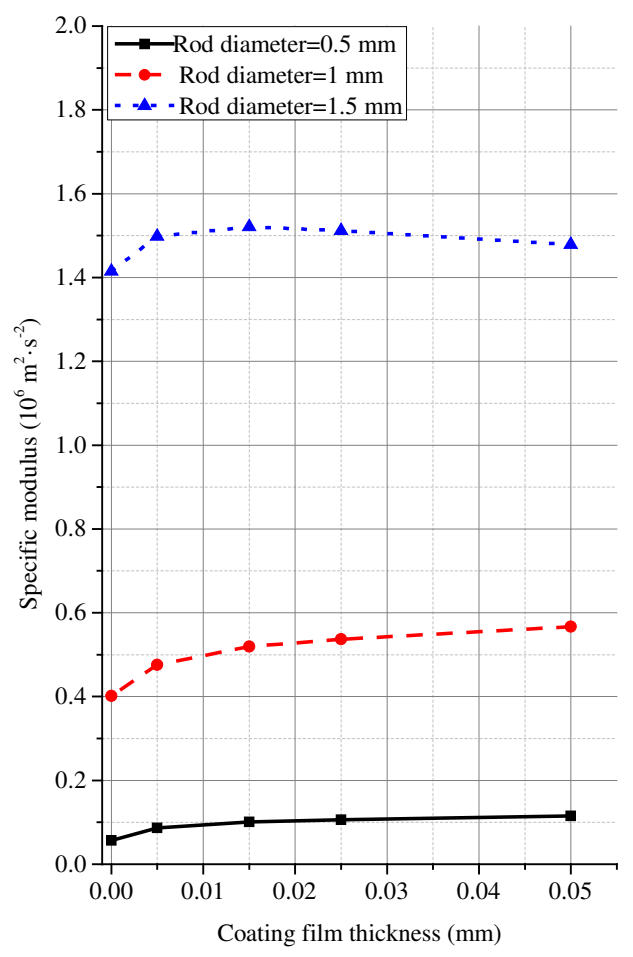

Fig. 15 Specific modulus of coated lattice unit

The specific modulus of lattice unit increases remarkably after the coating. However, when the thickness of coating film increased linearly, it does not lead to a visible positive impact. Overall, there should be an optimal coating thickness for the maximum stiffnessweight-ratio of lattice structure. And for the BCC lattice sample in this work, $15 \mu \mathrm{m}$ could be a recommend value.

\section{CONCLUSIONS}

A multiscale evaluation of mechanical properties for BCC metal coated lattice structures is well studied in this work. The achievements can be highlighted as follows:

(1) The presented numerical Approach 1 and 2, both are established based on the homogenization idea, while the former one used the volume weighted stress, and the latter one used the reaction force in stress calulation. For lattice structure, the latter one is slightly more accurate.

(2) For lattice homogenization, the multiple cubic units are recommended as the RVE for the numerical evaluation.

(3) The increase of volume fraction, also represented by the rod diameter, has a great impact on the homgenized elastic modulus and specific modulus.

(4) The coating film may remarkably increase the stiffness and specific modulus of the lattice structures. However, there should be an optimal coating thickness for the maximum stiffness-weight-ratio of lattice structure.

(5) Impact of coating parameters and 3D printing parameters will be further studied to provide more reliable design reference for the lattice structures.

\section{DECLARATION}

\section{Funding}

Supported by National Natural Science Foundation of China (Grant No. 51805447, 61504024), XJTLU Key Program Special Fund (Grant No. KSF-E-01, KSF-E-27) and Research Development Fund (RDF-17-02-44), Open Project of Railway Vehicle Engineering（BMRV20KF03）

Availability of data and materials

The datasets supporting the conclusions of this article are included within the article.

\section{Authors' contributions}

The author' contributions are as follows: ** was in charge of the whole trial; ** wrote the manuscript; $* *$ assisted with sampling and laboratory analyses.

Competing interests

The authors declare no competing financial interests. 
Novel 6-DOF Wearable Exoskeleton Arm with Pneumatic Force-Feedback for Bilateral Teleoperation

Consent for publication

Not applicable

Ethics approval and consent to participate

Not applicable

\section{References}

[1] S. Soleimanian, A. Davar, R. Azarafza, J. E. Jam, and M. R. Zamani, "Theoretical, numerical, and experimental analyses of free vibrations of glass fiber reinforced polymer plates with central cutouts and free boundaries," Mech. Adv. Compos. Struct., vol. 5, no. 1, pp. 67-74, 2018.

[2] M. Azhari, A. R. Shahidi, and M. M. Saadatpour, "Local and post local buckling of stepped and perforated thin plates," Appl. Math. Model., vol. 29, no. 7, pp. 633-652, 2005.

[3] H. Takabatake, "Static analysis of elastic plates with voids," Int. J. Solids Struct., vol. 28, no. 2, pp. 179-196, 1991.

[4] R. M. Gorguluarslan, U. N. Gandhi, R. Mandapati, and S. K. Choi, "Design and fabrication of periodic lattice-based cellular structures," Comput. Aided. Des. Appl., vol. 13, no. 1, pp. 50-62, 2016.

[5] D. Fabris et al., "Mechanical properties of zirconia periodic open cellular structures," Ceram. Int., vol. 45, no. 13, pp. 15799-15806, 2019.

[6] T. D. Ngo, A. Kashani, G. Imbalzano, K. T. Q. Nguyen, and D. Hui, "Additive manufacturing (3D printing): A review of materials, methods, applications and challenges," Compos. Part B Eng., vol. 143, no. December 2017, pp. 172-196, 2018.

[7] R. Xiao et al., "3D printing of titanium-coated gradient composite lattices for lightweight mandibular prosthesis," Compos. Part B, vol. 193, no. January, p. 108057, 2020.

[8] M. Matthew, M. Jurg, M. Leary, and M. Brandt, "Programmatic Lattice Generation for Additive Manufacture," 2015, vol. 20, no. July, pp. 178-184.

[9] F. Tamburrino, S. Graziosi, and M. Bordegoni, "The design process of additively manufactured mesoscale lattice structures: A review," J. Comput. Inf. Sci. Eng., vol. 18, no. 4, pp. 1-16, 2018.

[10] C. Yanhong, Z. Weimin, W. Shiwen, L. I. N. Jianguo, B. Daniel, and S. Debin, "Investigation of FE Model Size Definition for Surface Coating Application CDM-based Constitutive Model," CHINESE J. Mech. Eng., vol. 25, no. 5, pp. 860-867, 2012.

[11] M. Jimenez, S. Duquesne, and S. Bourbigot, "Intumescent fire protective coating: Toward a better understanding of their mechanism of action," Thermochim. Acta, vol. 449, no. 1-2, pp. 16-26, 2006.

[12] Y. Kojima, T. Aizawa, and S. Kamado, "Umehara-2000-
Mat Sci Forum-Structure and Corrosion Behavior of Conversion Coatings on Magnesium Alloys." pp. 273282, 2000.

[13] C. T. Kao, C. C. Lin, Y. W. Chen, C. H. Yeh, H. Y. Fang, and M. Y. Shie, "Poly(dopamine) coating of 3D printed poly(lactic acid) scaffolds for bone tissue engineering," Mater. Sci. Eng. C, vol. 56, pp. 165-173, 2015.

[14] J. Song et al., "Metal-coated hybrid meso-lattice composites and their mechanical characterizations," Compos. Struct., vol. 203, no. November 2017, pp. 750 763, 2018.

[15] K. B. Yilmaz, Çömez, M. A. Güler, and B. Yildirim, "Analytical and finite element solution of the sliding frictional contact problem for a homogeneous orthotropic coating-isotropic substrate system," ZAMM Zeitschrift fur Angew. Math. und Mech., vol. 99, no. 3, pp. 1-17, 2019.

[16] R. Jahedi, S. Adibnazari, and G. H. Farrahi, "Performance Analysis of Functionally Graded Coatings in Contact with Cylindrical Rollers," Adv. Mech. Eng., vol. 7, no. 1, 2015.

[17] B. Sobol, A. Soloviev, and A. Krasnoschekov, "The transverse crack problem for elastic bodies stiffened by thin elastic coating," ZAMM Zeitschrift fur Angew. Math. und Mech., vol. 95, no. 11, pp. 1302-1314, 2015.

[18] Z. Gaimei, H. E. Cunfu, W. U. Bin, and C. Qiang, "Evaluating Local Elasticity of the Metal Nano-films Quantitatively Based on Referencing Approach of Atomic Force Acoustic Microscopy," CHINESE J. Mech. Eng., vol. 25, 2012.

[19] E. Ptochos and G. Labeas, "Elastic modulus and Poisson's ratio determination of micro-lattice cellular structures by analytical, numerical and homogenisation methods," J. Sandw. Struct. Mater., vol. 14, no. 5, pp. 597-626, 2012.

[20] S. Kurukuri, "A Review of Homogenization Techniques for Heterogeneous Materials,” Analysis, p. 46, 2004.

[21] C. Li, L. Chen, and L. Qiao, "RVE Based Numerical Evaluation on Effective Mechanical Properties of Composite with Randomly Distributed Multi-Phase Inclusions," Adv. Mater. Res., vol. 383-390, pp. 931-934, 2012.

[22] M. Ostoja-Starzewski, "Material spatial randomness: From statistical to representative volume element," Probabilistic Eng. Mech., vol. 21, no. 2, pp. 112-132, 2006.

[23] N. Carrere, R. Valle, T. Bretheau, and J. L. Chaboche, "Multiscale analysis of the transverse properties of Tibased matrix composites reinforced by SiC fibres: From the grain scale to the macroscopic scale," Int. J. Plast., vol. 20, no. 4-5, pp. 783-810, 2004.

[24] K. Okomori, T. Enomae, and F. Onabe, "Evaluation and control of coated paper stiffness," 1999, vol. 132, pp. 121-132.

[25] H. W. Ng and Z. Gan, "A finite element analysis technique for predicting as-sprayed residual stresses generated by 
the plasma spray coating process," Finite Elem. Anal. Des., vol. 41, no. 13, pp. 1235-1254, 2005.

[26] I. Bencheikh, F. Bilteryst, and M. Nouari, "Modelling of the thermomechanical behaviour of coated structures using single and multi-level-set techniques coupled with the eXtended Finite Element Method," Finite Elem. Anal. Des., vol. 134, no. June, pp. 68-81, 2017.

[27] P. M. Dixit and S. D. Uday, Plasticity Fundamentals and Applications. CRC Press, 2015.

[28] I. T. Zohdi and P. Wiggers, "An introduction to computational micromechanics." Springer Science \& Business Media, 2008.

[29] Y. K. Khdir, T. Kanit, F. Zaïri, and M. Naït-Abdelaziz, "Computational homogenization of plastic porous media with two populations of voids," Mater. Sci. Eng. A, vol. 597, pp. 324-330, 2014.

[30] M. Chen and A. Hachemi, "Progress in Plastic Design of Composites," in Direct Methods for Limit States in Structures and Materials, 2014, pp. 119-138.

[31] J. Li, Y. Wang, G. Xiang, H. Liu, and J. He, "Hybrid Additive Manufacturing Method for Selective Plating of Freeform Circuitry on 3D Printed Plastic Structure," Adv. Mater. Technol., vol. 4, no. 2, pp. 1-10, 2019.

[32] J. Li, Y. Wang, P. Wang, J. He, H. Liu, and G. Xiang, "Rapid production of customized 3D electronics via hybrid additive manufacturing technology," Proc. Electron. Components Technol. Conf., vol. 2019-May, pp. 135-140, 2019.

[33] T. Sanderson, "Measuring the elastic moduli of electroless nickel-phosphorus deposits," Plat. Surf. Finish., vol. 92, no. 6, pp. 39-43, 2005.

[34] K. Refai et al., "Determination of the effective elastic properties of titanium lattice structures Determination of the effective elastic properties of titanium lattice structures," Mech. Adv. Mater. Struct., vol. 0, no. 0, pp. 1$14,2019$.

\section{Biographical notes}

Sina Soleimanian, born in 1991, is currently a PhD candidate of Xi' an Jiaotong-Liverpool University (XJTLU) and University of Liverpool. He received his bachelor and master degrees in Mechanical Engineering and Composite Materials Engineering both from Iran.

Tel: $\quad$ +86-13073317304; $\quad$ E-mail: s.soleimanian18@student.xjtlu.edu.cn

Xiang Wang, born in 1990, is currently a PhD candidate Xi' an Jiaotong-Liverpool University (XJTLU) and University of Liverpool. He received his bachelor degree in Environmental Engineering. $\quad$ Tel: +86-15056935772; $\quad$ E-mail: xiang.wang@xjtlu.edu.cn
Min Chen, born in 1981, is currently an associate professor at Xi' an Jiaotong-Liverpool University (XJTLU).

Tel: +86-88167131; E-mail: min.chen@xjtlu.edu.cn

Yanqing Yu, born in 1995, is currently a MSc candidate at Southeast University (SEU). She received her bachelor degree in electronic science and technology.

Ji Li, born in 1983, is currently an associate professor at Southeast University (SEU). Email: j.li5@ @eu.edu.cn

Geng Chen, born in 1985, is currently a professor at Beijing Jiaotong University (SEU). Email: gengchen@bjtu.edu.cn 


\section{Figures}
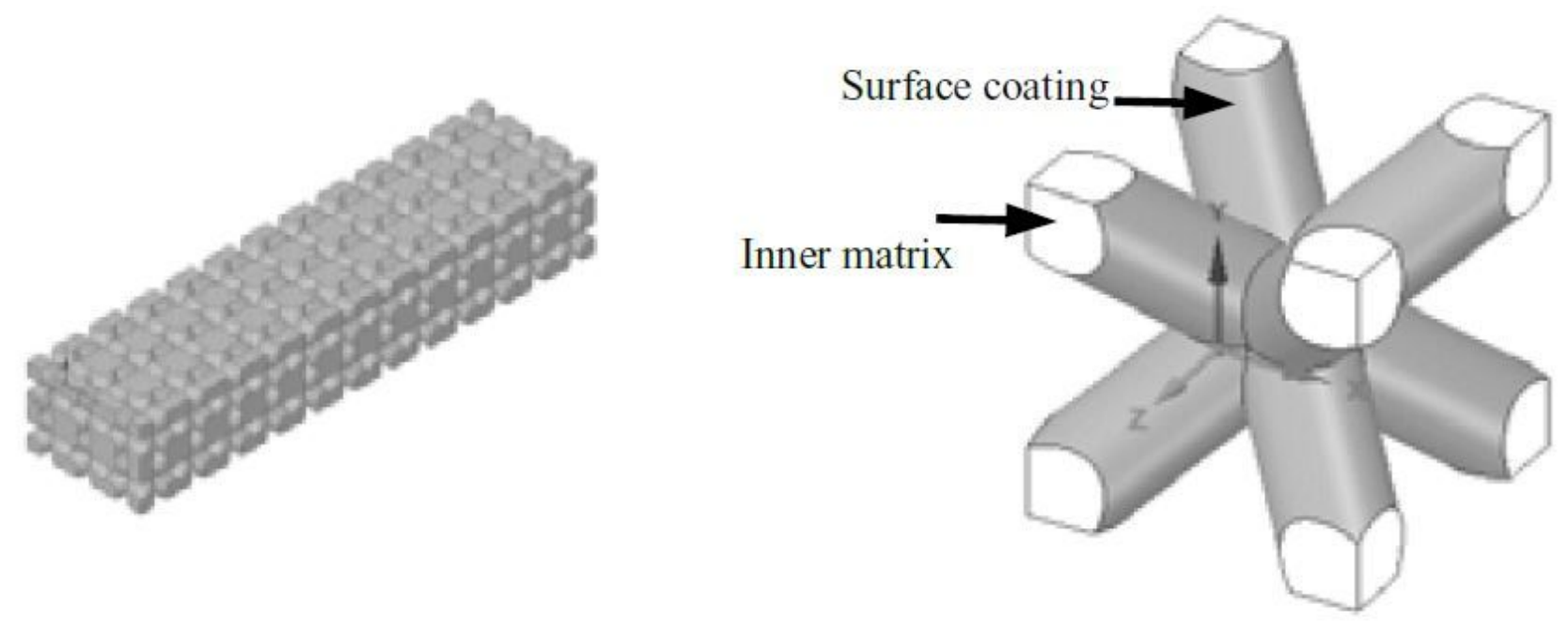

\section{Figure 1}

BCC-like lattice structure modeling at: (a) global level, (b) RVE level.
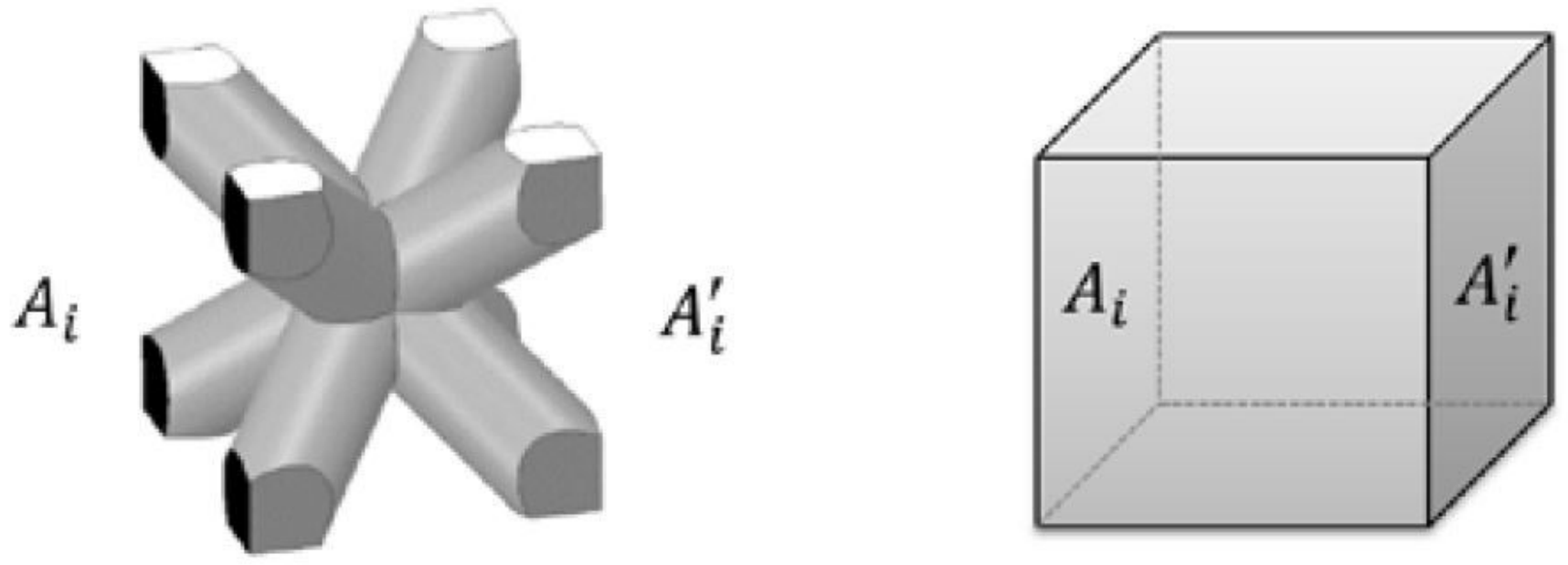

Figure 2

Periodic boundary condition: (a) Lattice unit cell, (b) Equivalent lattice unit 

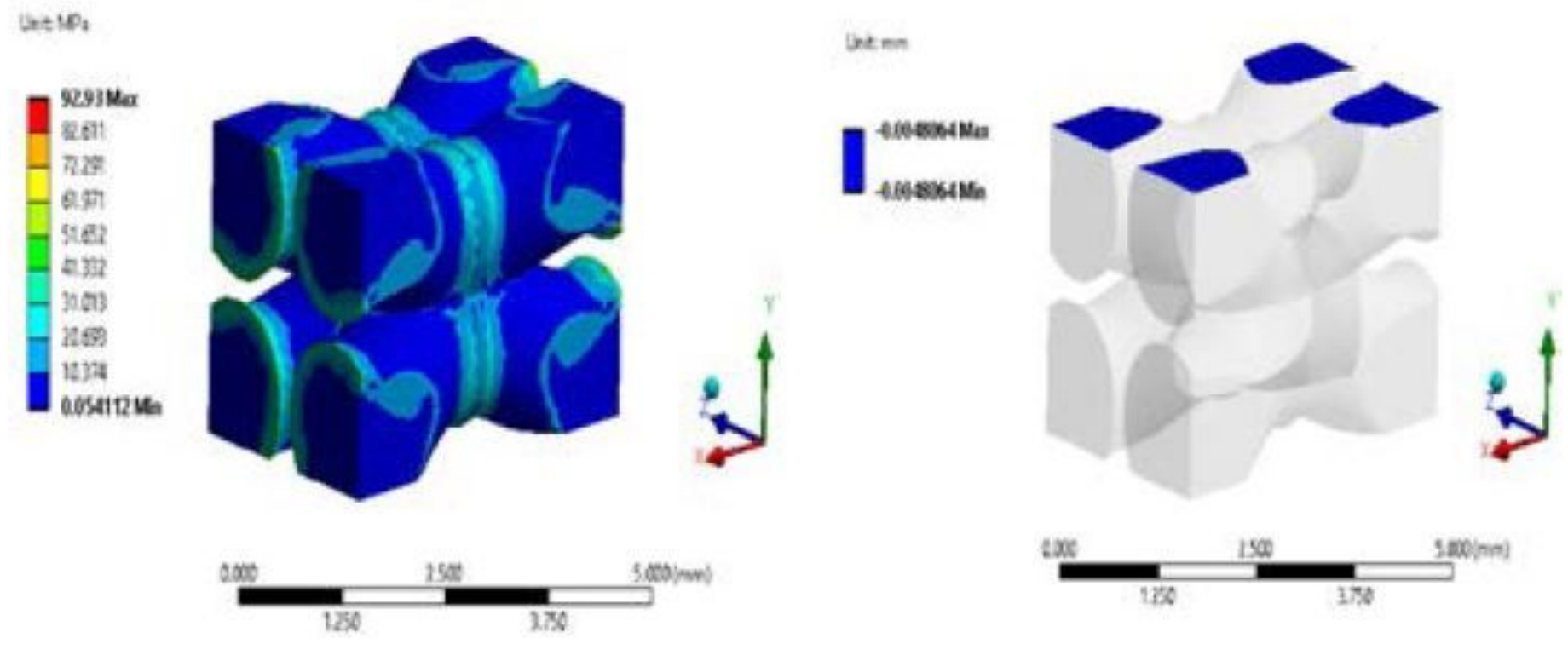

Figure 3

(a) stress contour, and (b) transverse displacement contour for the lattice RVE

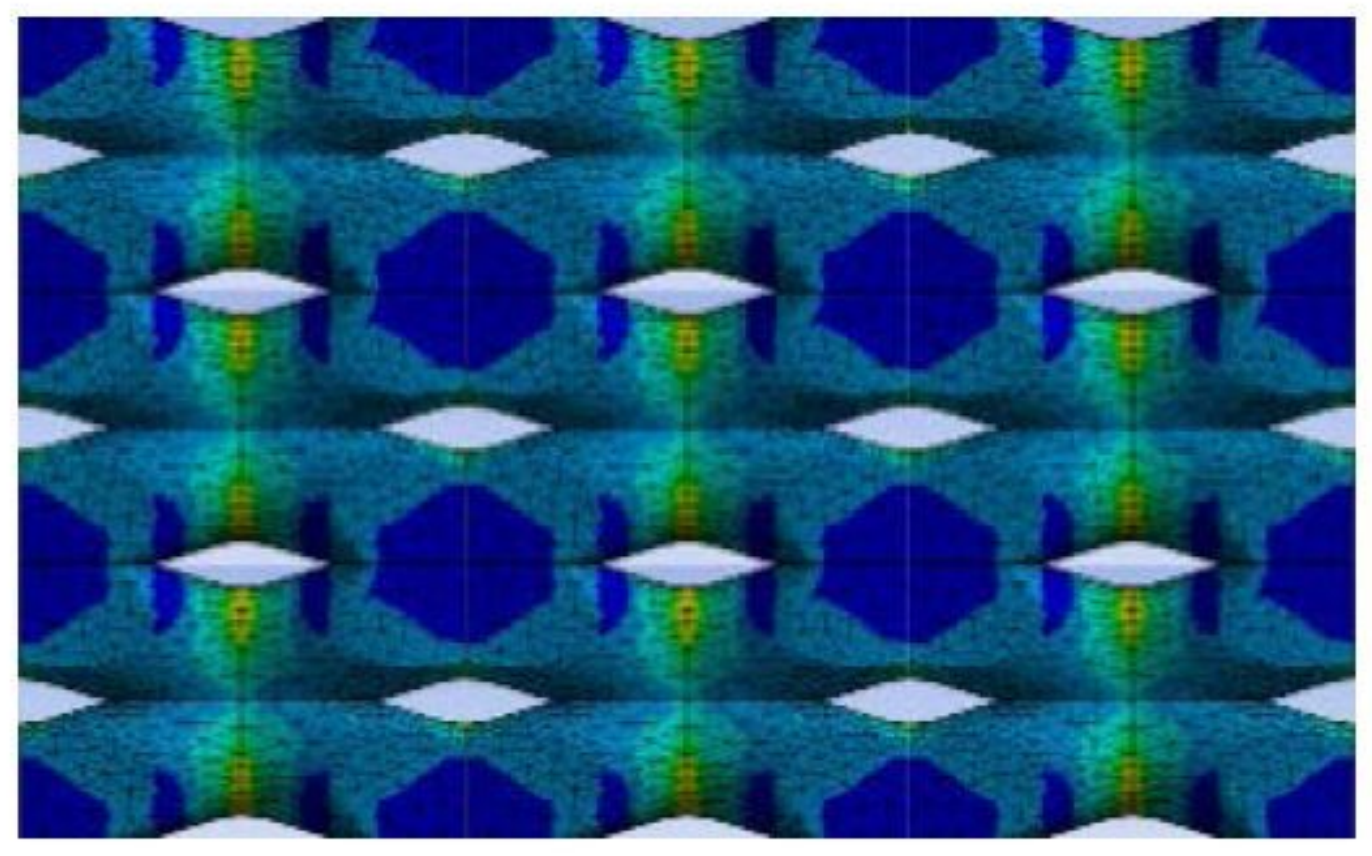

Figure 4

Stress field of expanded deformed RVE 


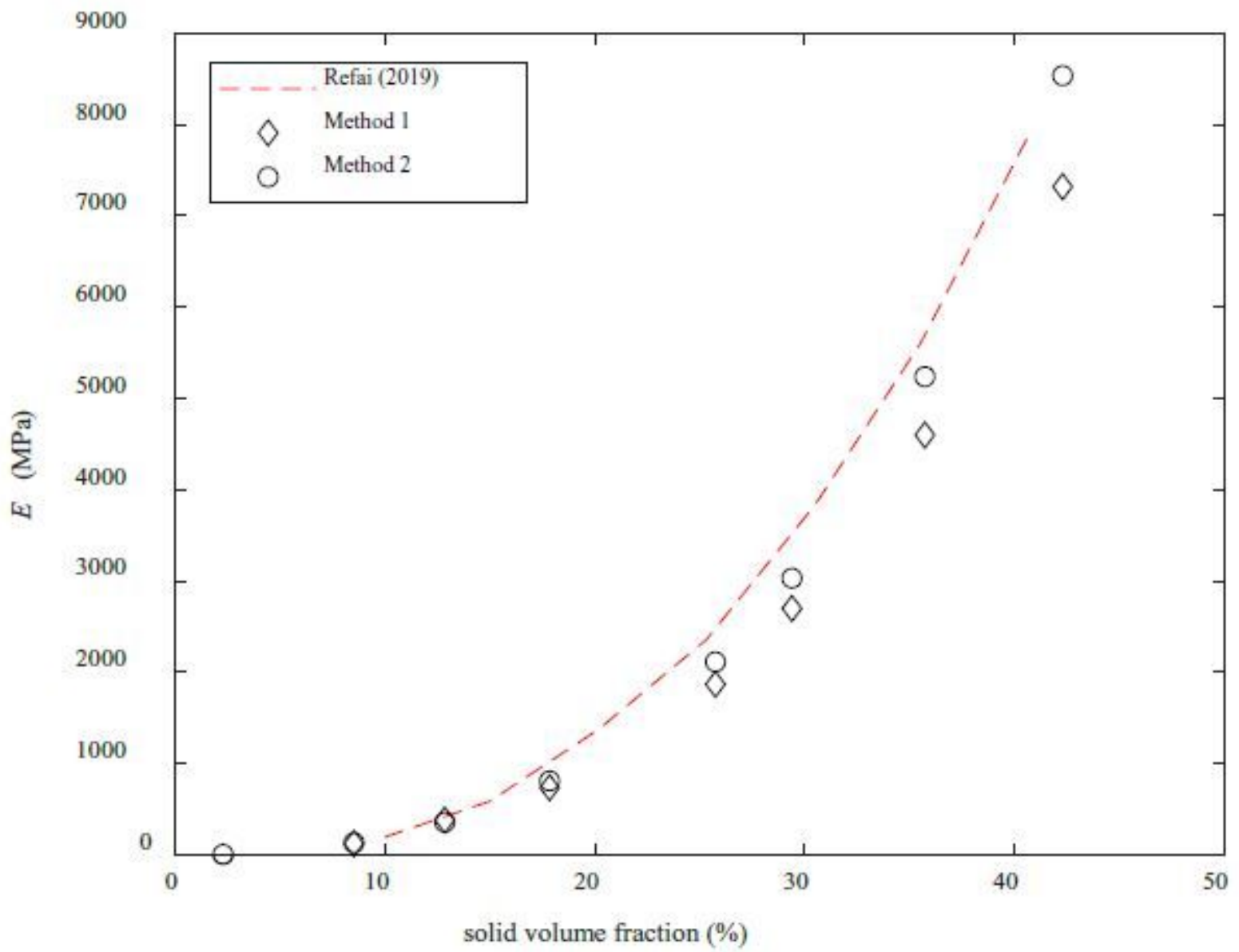

Figure 5

Comparison of effective elastic modulus for BCC lattice RVE with various rod diameters.

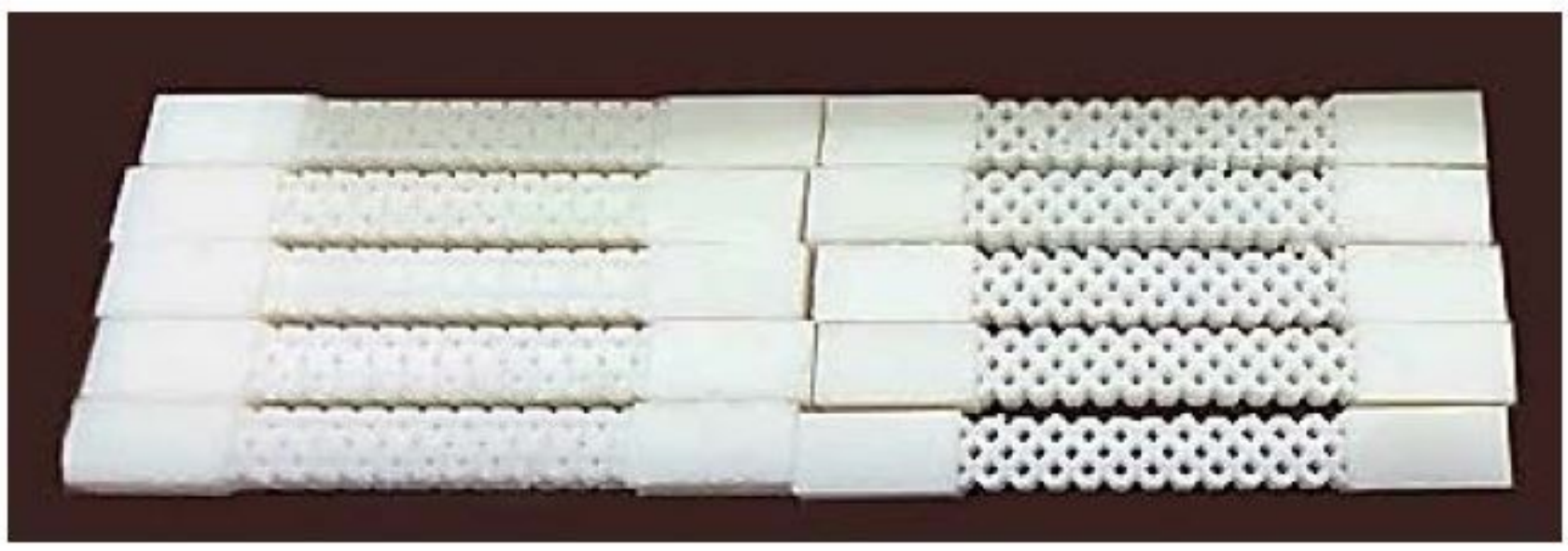

Figure 6

3D printed lattice samples 


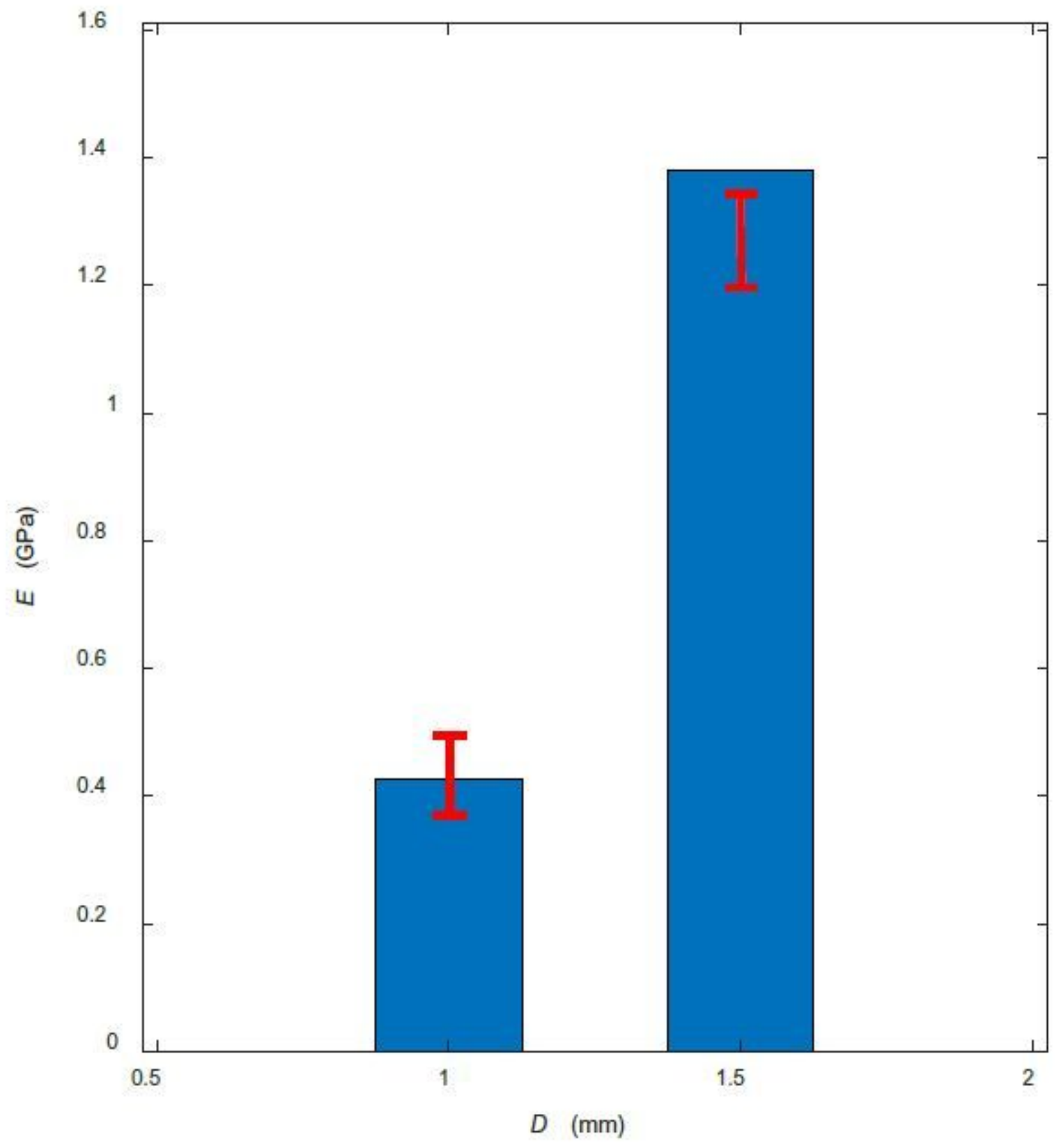

Figure 7

Validation of numerical modeling with experiment evaluating the effective elastic modulus for lattice structure 

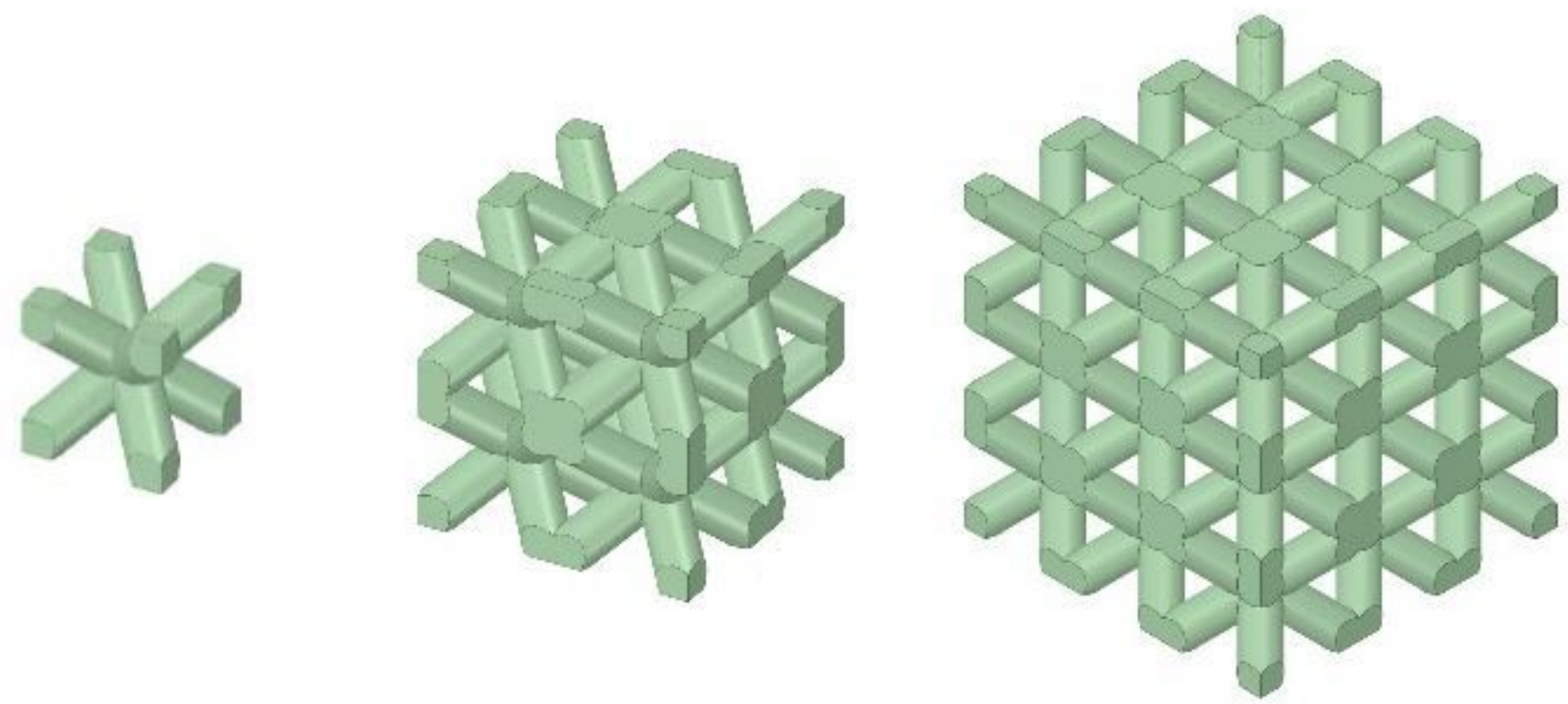

$1 \times 1 \times 1$

$2 \times 2 \times 2$

$3 \times 3 \times 3$

Figure 8

Three RVE definitions.

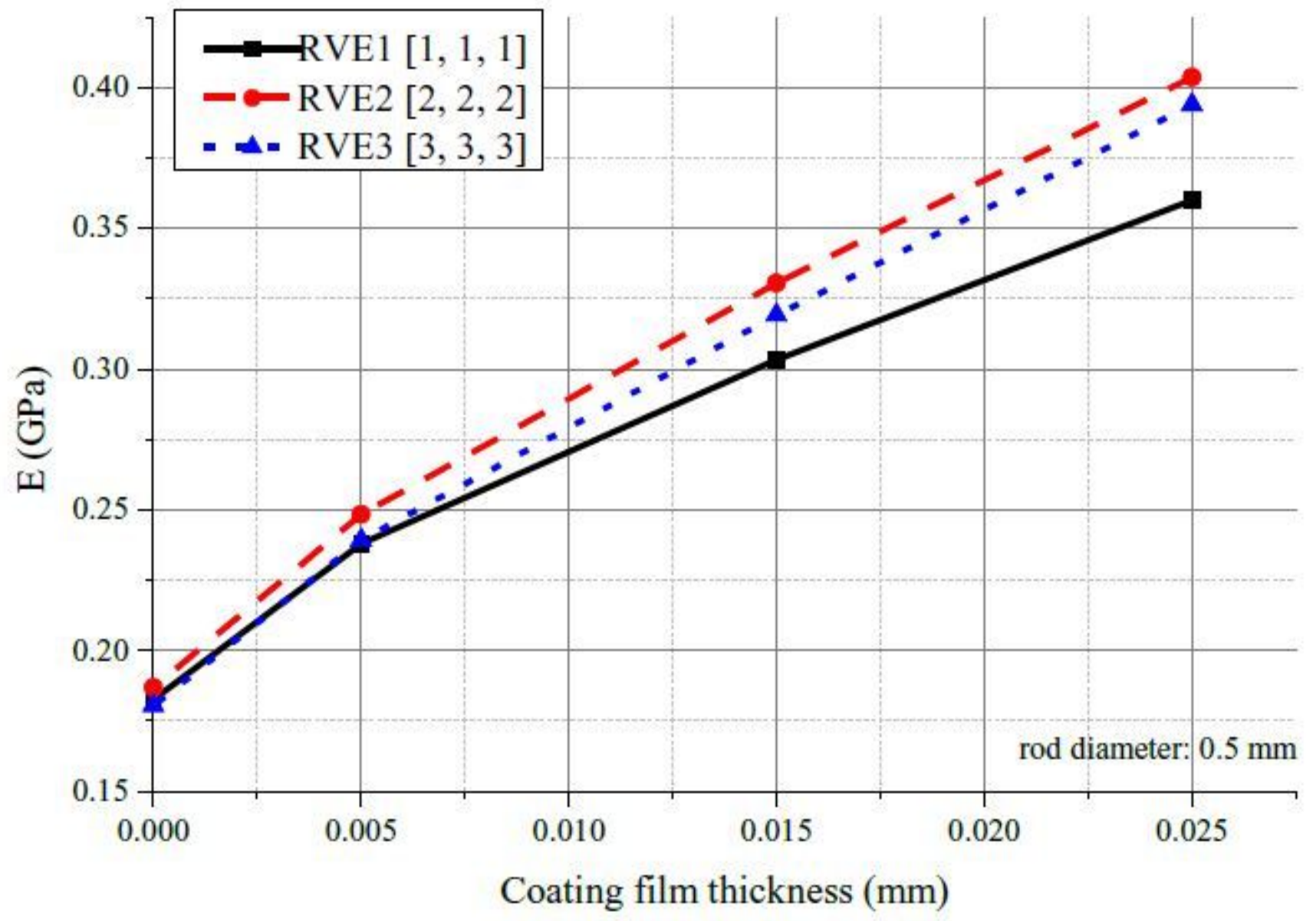

Figure 9 
Effective Young's modulus for a BCC lattice with different number of unit cells $(\mathbb{\nabla}=0.5 \mathrm{~mm})$

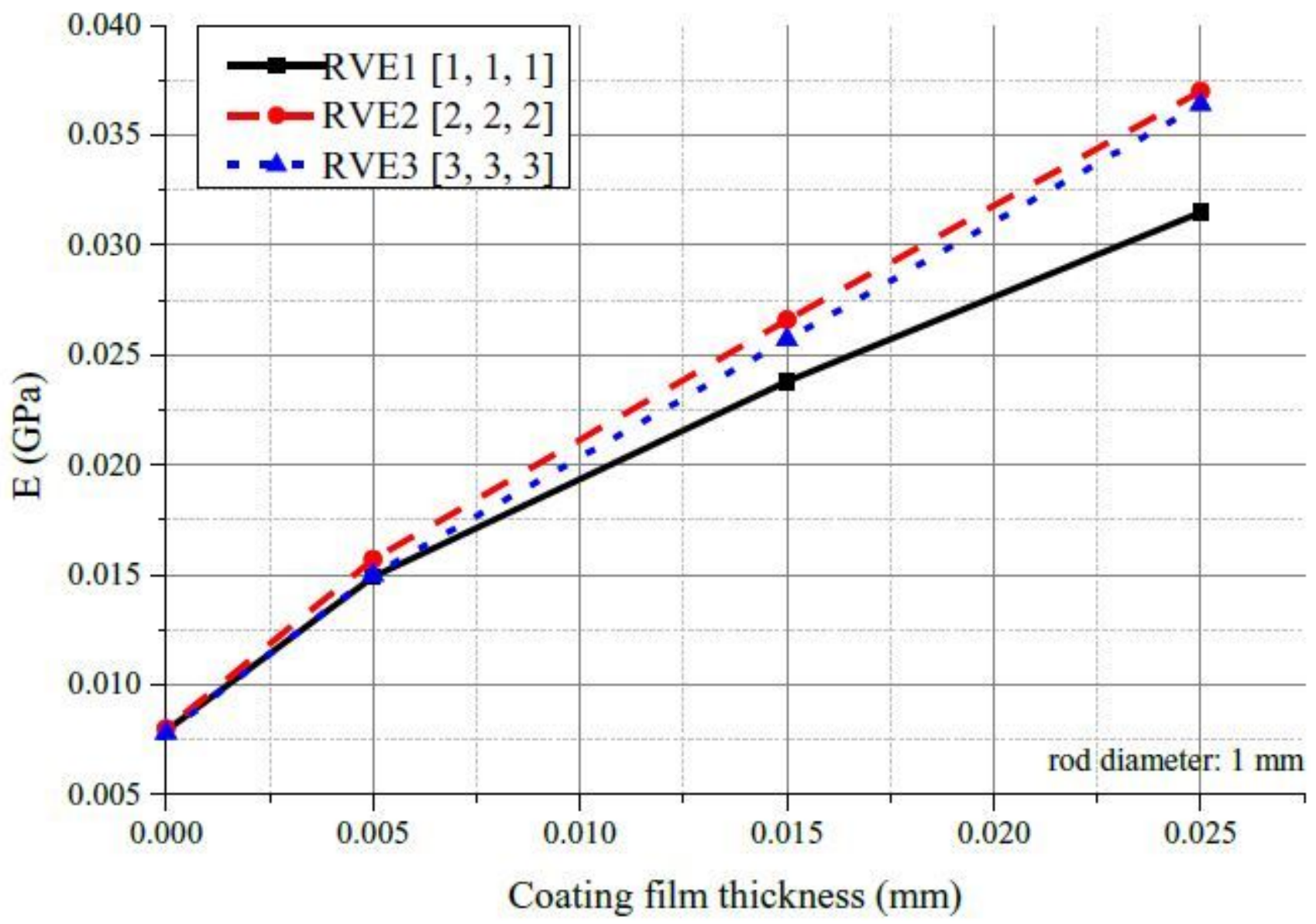

Figure 10

Effective Young's modulus for a BCC lattice with different number of unit cells $(\mathbb{Q} \mathbb{Q}=1.0 \mathrm{~mm}$ ) 


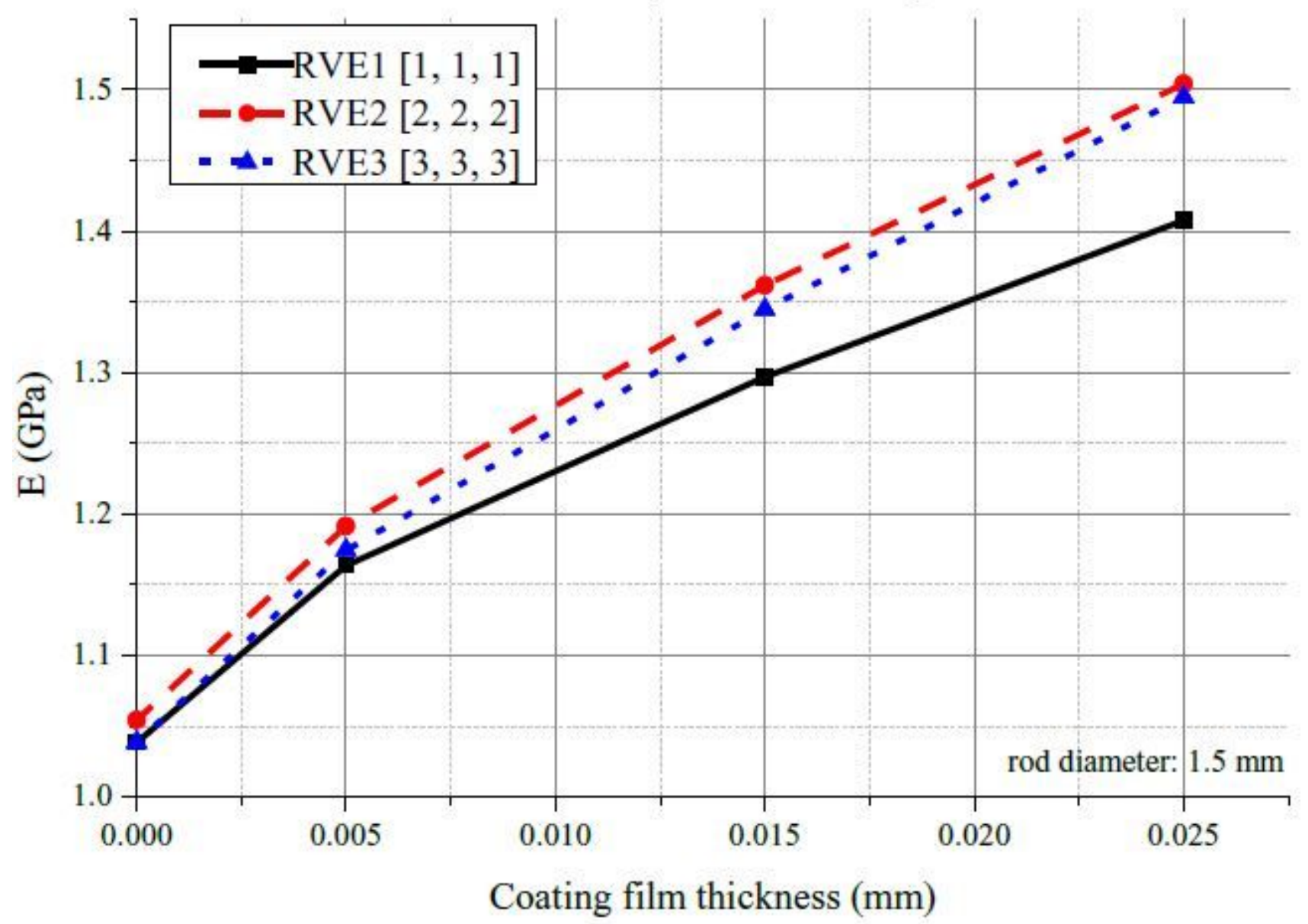

Figure 11

Effective Young's modulus for a BCC lattice with different number of unit cells $(\mathbb{Q}=1.5 \mathrm{~mm})$ 


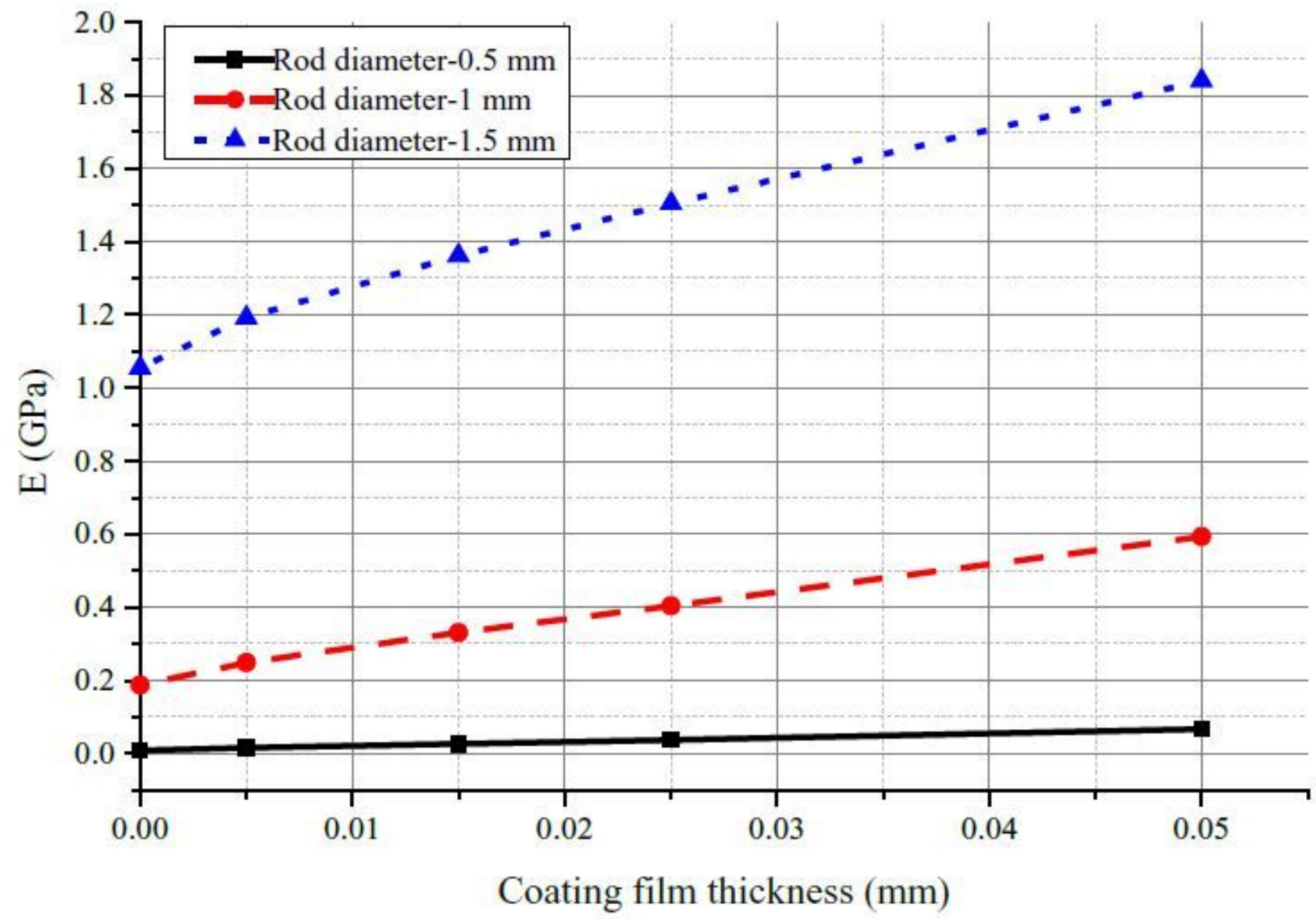

Figure 12

Effective Young's modulus for a BCC lattice with different diameter of unit cells and coating film thickness (RVE [2,2,2] selected). 


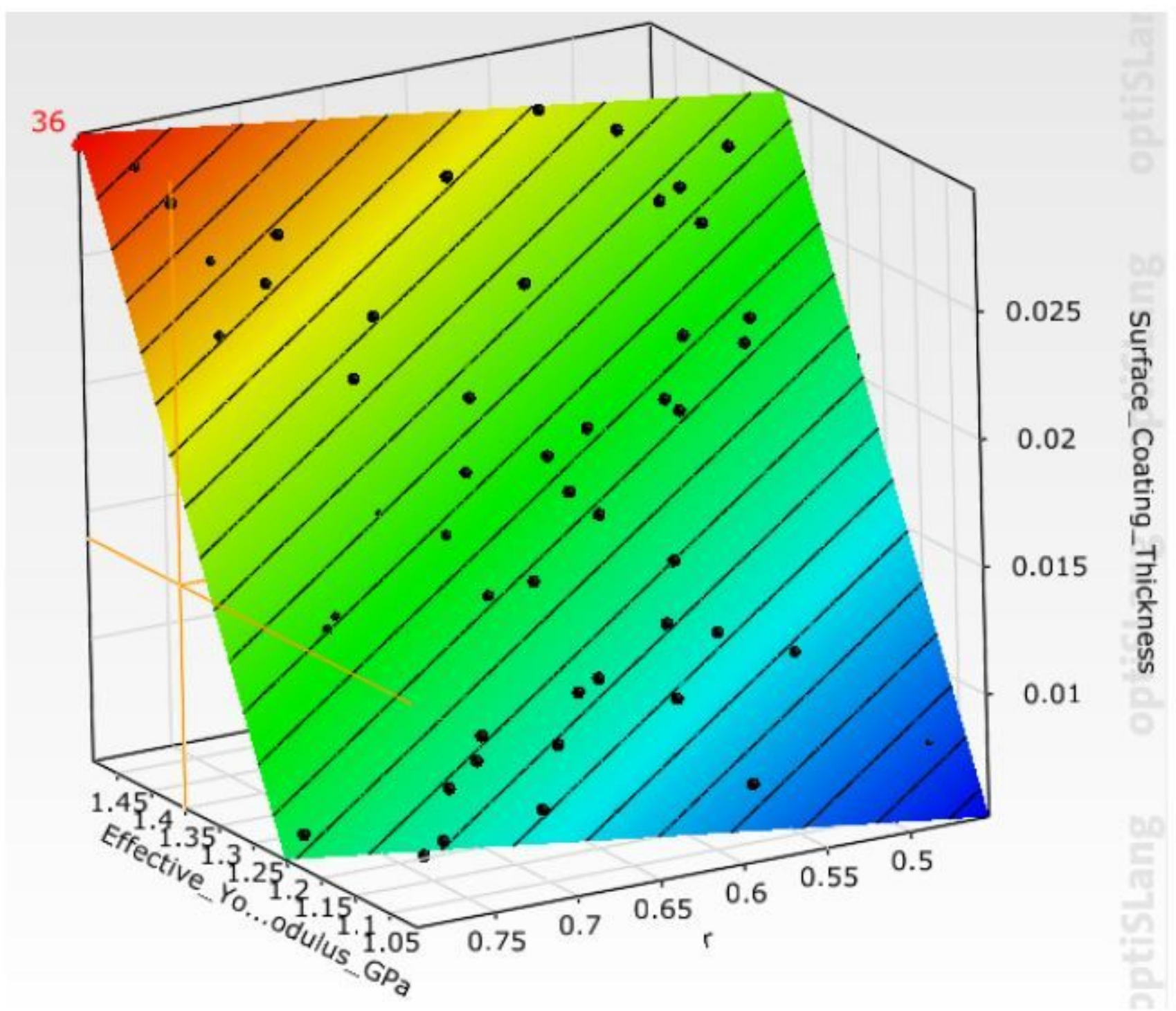

Figure 13

Overall impact of rod radius and coating thickness on effective elastic modulus 


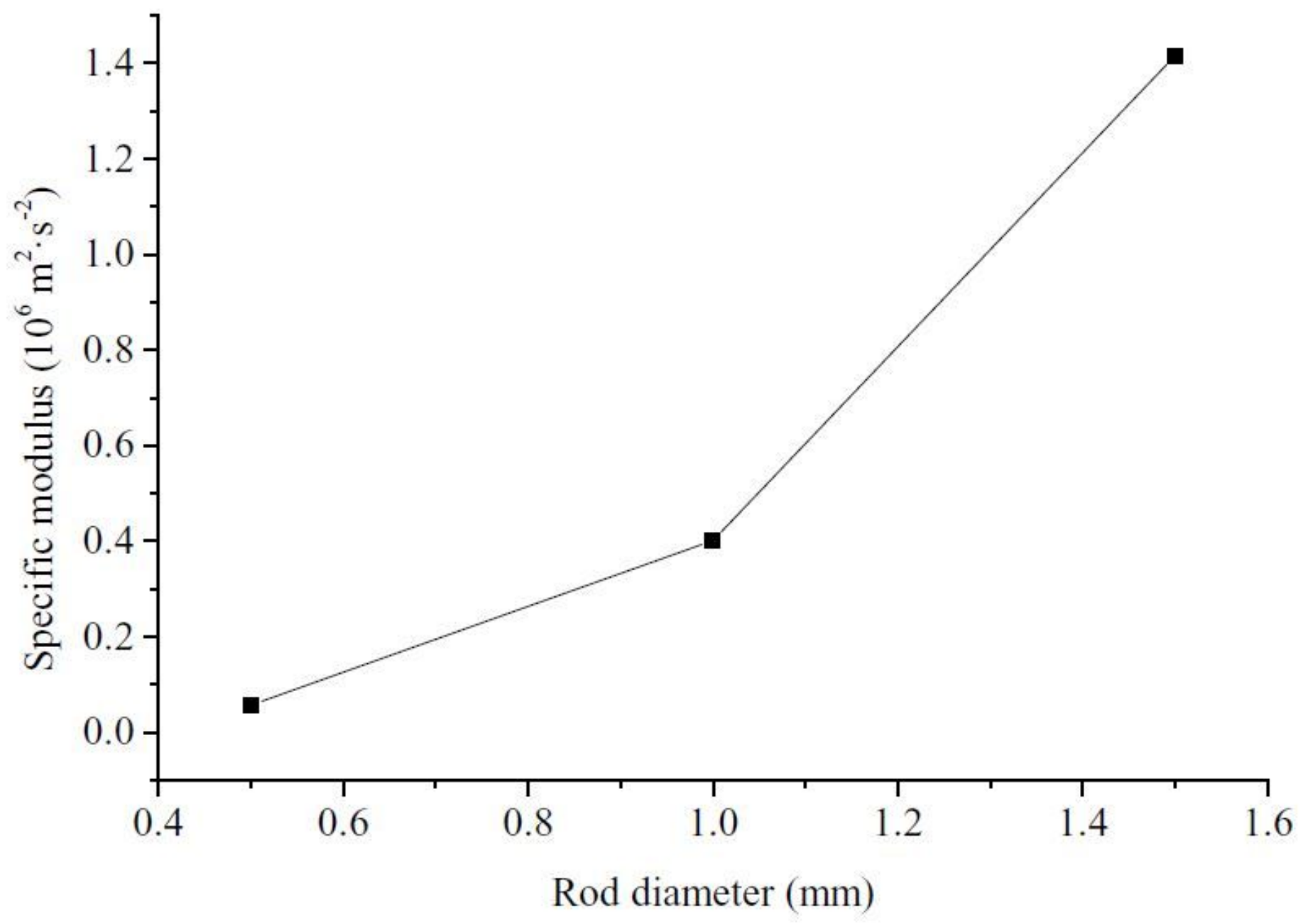

Figure 14

Specific modulus of pure lattice units 


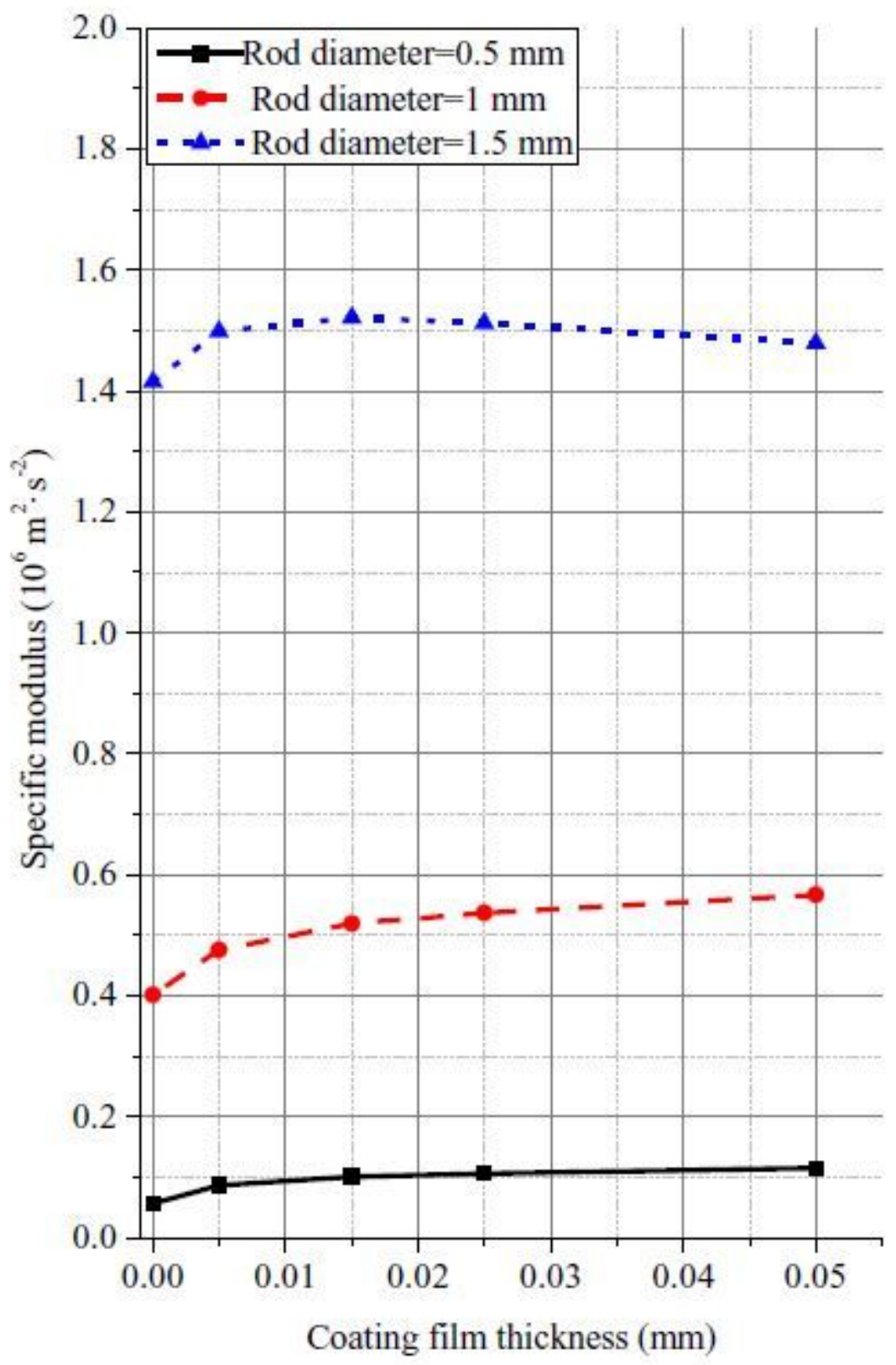

Figure 15

Specific modulus of coated lattice unit 